\title{
Planes padrino en instituciones como mecanismo de deserción
}

\section{Planos padrinho em instituições como mecanismo de deserção}

\author{
Gladys Amanda Portilla-Peña ${ }^{1}$, Yuleima Agudelo-González²
}

Forma de citar: Portilla-Peña, G., \& Agudelo-González, Y. Planes padrino en instituciones como mecanismo de deserción. Revista Eco.Mat. 7 [141-148].

Recibido:

Agosto 10 de 2015

Aceptado:

Diciembre 6 de 2015

\section{Resumen}

La investigación se desarrolló en el marco de la investigación cuantitativa, correlacional, explicativa. Su objetivo fue analizar el proyecto "plan padrino" con el sector productivo de la región para estimular el apoyo económico a estudiantes en riesgo de deserción. Resultados: un $50 \%$ de los jóvenes que ingresan por primera vez y son apadrinados desertan por otras causas. La deserción disminuyó por año apoyada de factores académicos. Conclusión: El plan padrino permitió estudiar entre 1 y 4 jóvenes por semestre, no tuvo relevancia significativa en la deserción de las instituciones de educación superior.

Palabras clave: apoyo económico, deserción, Plan padrino.

\begin{abstract}
The research was developed within the framework of quantitative, correlational, explanatory research. Its objective was to analyze the project "padrino plan" with the productive sector of the region to stimulate the economic support to students at risk of desertion. Results: $50 \%$ of the young people who enter for the first time and are sponsored deserted for other causes. Dropout declined each year supported by academic factors. Conclusion: The sponsor plan made it possible to study between 1 and 4 young people per semester. It did not have significant relevance in the desertion of higher education institutions.
\end{abstract}

keywords: Dropout, financial support, Sponsor plan.
${ }^{1}$ Doctorado en Educación. Directiva

amandaportilla70@gmail.com Docente Institución Educativa Julio Pérez Ferrero. Cúcuta-Colombia

${ }^{2}$ Doctorado en Educación memita310221@gmail.com Docente Institución Educativa Mercedes Abrego Cúcuta-Colombia

\section{Resumo}

Este estudo foi realizado no âmbito da pesquisa quantitativa, correlacional e explicativa. Seu objetivo foi analisar o projeto "plano padrinho" com o sector produtivo da região para estimular o apoio económico a estudantes em risco de deserção. Resultados: um $50 \%$ dos jovens que ingressaram pela primeira vez e foram apadrinhados desertaram por outras causas. A deserção diminuiu por ano apoiada de fatores acadêmicos. Conclusão: $\mathrm{O}$ plano padrinho permitiu estudar entre 1 e 4 jovens por semestre, e mostrou que não teve relevância significativa na deserção das instituições de educação superior.

Palavras-chave: apoio financeiro, deserção, Plano padrinho. 
No. 1

\section{Introducción}

En el Departamento, las instituciones de educación básica y universidades han establecido relación directa con el sector empresarial de la región para llevar a cabo el programa de apadrinamiento básico (de uno a cinco), plus (de cinco a diez) y máster (de 10 a 20) jóvenes que ingresan en condiciones socio económicas precarias a realizar un pregrado en la institución y estudiantes que se encuentren en riesgo de deserción por factor socio económico en instituciones de educación (Reyes, Castañeda \& Pabón, 2012), donde el apoyo económico sea dado desde el ingreso, el desarrollo y la culminación de una carrera profesional que ofrece la institución, permitiéndole un espacio dentro de una comunidad educativa donde logre construir competencias básicas primordiales para que a futuro obtenga una vida exitosa en todos los campos y donde el principal reto institucionalempresarial sea el de formar profesionales de cambio que requiere el país Wilson (2011).

Fue tarea de los gestores de las instituciones, crear los acuerdos y convenios necesarios con el sector empresarial donde el acompañamiento y apoyo a los estudiantes en riesgo de deserción por factor económico tengan el reconocimiento institucional público (Huertas, Esmeral \& Sánchez, 2014) y posibiliten al estudiante cubrir sus estudios y en contraprestación este realice su práctica profesional con horarios flexibles y disponibles tanto para la empresa como para que el estudiante continúe cumpliendo con sus responsabilidades académicas y como resultado final desarrolle un proyecto ya sea de investigación o aplicación beneficiando la productividad, los procesos o productos de dicha empresa apadrinadora.

La motivación dada al sector empresarial según Wilson (2012) y Wood (2010) en cada periodo académico se dio constantemente con la firme intencionalidad de vincularla a los programas que ofrece la institución y las necesidades de la población que soliciten apadrinamiento, exaltada y reconocida por la institución por el apoyo que brinde a la formación integral de los futuros profesionales que fortalecerán el mismo sector empresarial y productivo de la región.

La investigación buscó analizar el proyecto "plan padrino" con el sector productivo de la región para estimular el apoyo económico a estudiantes en riesgo de deserción. Proyecto en el cual se socializó el proyecto de Plan padrino con el sector empresarial de la región para disminuir la tasa de deserción en la institución (Martínez, Vergel \& Zafra, 2015) y brindar apoyo económico a los estudiantes desde el inicio y en riesgo de deserción, se vincular el sector empresarial de la región con la academia para el desarrollo del programa plan padrino, se buscó motivar al sector empresarial para apadrinar estudiantes en riesgo de deserción, permitiéndole desarrollar sus competencias laborales y profesionales en contraprestación al apoyo económico Wilson y Blankenship, (2010), con trabajos de grado en investigación o aplicación que beneficien la empresa. Mantener y dar continuidad en cada periodo el plan padrino dándolo a conocer a todo el sector empresarial de la región y comunidad académica.

\section{Metodología}

La investigación sigue un enfoque cuantitativo, explicativo, diseño Correlacional Martínez, Vergel \& Zafra, (2015). Así mismo se analiza desde un diseño holístico proyectivo "este tipo de investigación (la Investigación Proyectiva), también llamado proyecto factible, consiste en la elaboración de una propuesta o de un modelo, como solución a un problema o necesidad de tipo práctico Acevedo, Vergel \& Flórez (2012), ya sea de un grupo social, o de una institución, en un área particular del conocimiento, a partir de un diagnóstico preciso de las necesidades del momento Turizo (2014), los procesos explicativos o generadores involucrados y las tendencias futuras Rincón(2011). También se pueden ubicar como proyectivas, todas aquellas 
investigaciones que conducen a inventos, programas, diseños o a creaciones dirigidas a cubrir una determinada necesidad, y basadas en conocimientos anteriores. Según Vergel, Martínez, Zafra (2015), la invención consiste en hallar solución a los problemas prácticos encontrando nuevas formas e instrumentos de actuación y nuevas modalidades de su aplicación en la realidad”. Hurtado, (2000)

Se realiza análisis descriptivo de variable número de estudiantes que han recibido ayuda por plan padrino. Se realizan pruebas de hipótesis chi cuadrado y análisis de correlación. Estadística aplicada busca crear modelos econométricos, factoriales, diseños de experimentos Zafra, Martínez \& Vergel (2014); Isaac (2000), y matemática aplicada busca generar modelos serie de tiempo para modelar. Vergel, Martínez, \& Duarte (2015).

\section{Resultados}

En torno a la edad del aspirante aumenta la probabilidad de ser admitido, mientras que la edad al cuadrado la disminuye, no es un efecto lineal. Según estudio análisis de supervivencia Vergel, Martínez, Orjuela (2014), resultados de las funciones de supervivencia (cuartiles) muestran cómo el manejo de preconceptos en matemáticas biología, química como un factor que incrementa el riesgo de graduarse, los estudiantes de género masculino, así como aquellos que recibieron alguna sanción, presentan mayor tendencia al abandono Vergel, Gallardo \& Martínez, (2014). Los estudios de pregrado con una edad mayor o igual a los 30 años y casados e inferior a los 16 años tienen mayor riesgo de retiro o deserción, Los estudiantes que ingresan en programas de ingeniería aumentan el riesgo de graduarse. Cuatro determinantes fueron identificados como razones de riesgo a través del modelo de regresión de Cox, factores individuales, factores socioeconómicos, factores académicos y factores institucionales Vergel (2015).

En las instituciones públicas de educación superior se generó una política de retención y permanencia acorde a parámetros y políticas gubernamentales y firmas de acuerdo entre rectores de instituciones y el Ministerio de Educación Nacional.

Las actividades desarrolladas fueron:

- Reunión informativa sobre el proyecto a todos los gerentes, directivos de instituciones, padres de familia, profesores, benefactores de cada ciudad Vergel, Orjuela, \& Martínez (2014).

- Sensibilización a los estamentos administrativo y académico sobre la política para la retención y permanencia. Cárdenas, Vergel \& Rincón (2013)

- Se publicaron en ambientes web la política para la retención y permanencia.

En las tablas I y II se evidencia el número de apoyos financieros recibidos de las IES de los periodos 2009-1 hasta 2011-2 para estudiantes de la institución.

Tabla I. Registro de estudiantes beneficiarios de apoyo en spadies I semestre

\begin{tabular}{|c|c|c|c|c|c|c|}
\hline $\mathrm{N}^{\circ}$ de apoyos financieros recibidos de las IES & $2009-1$ & $2009-2$ & $2010-1$ & $2010-2$ & $2011-1$ & $2011-2$ \\
\hline Ninguno & $55,02 \%$ & $35,36 \%$ & $36,87 \%$ & $46,15 \%$ & $53,60 \%$ & $57,96 \%$ \\
\hline 1 & $9,70 \%$ & $24,13 \%$ & $28,16 \%$ & $24,85 \%$ & $22,59 \%$ & $20,72 \%$ \\
\hline 2 & $5,09 \%$ & $5,37 \%$ & $5,40 \%$ & $4,40 \%$ & $3,88 \%$ & $3,46 \%$ \\
\hline 3 & $7,02 \%$ & $6,28 \%$ & $5,21 \%$ & $4,59 \%$ & $3,92 \%$ & $3,32 \%$ \\
\hline 4 o más & $23,17 \%$ & $28,86 \%$ & $24,37 \%$ & $20,01 \%$ & $16,02 \%$ & $14,55 \%$ \\
\hline
\end{tabular}

Fuente: Universidades Spadies

Tabla II. Registro de estudiantes beneficiarios de apoyo en spadies II semestre

\begin{tabular}{|c|c|c|c|c|c|c|}
\hline $\mathrm{N}^{\circ}$ de créditos recibidos del ICETEX & $2009-1$ & $2009-2$ & $2010-1$ & $2010-2$ & $2011-1$ & $2011-2$ \\
\hline 1 & $25,00 \%$ & $26,27 \%$ & $27,04 \%$ & $30,56 \%$ & $32,21 \%$ & $32,35 \%$ \\
\hline 2 & $16,53 \%$ & $18,89 \%$ & $25,00 \%$ & $21,11 \%$ & $23,49 \%$ & $25,00 \%$ \\
\hline 3 & $16,95 \%$ & $16,59 \%$ & $15,31 \%$ & $16,11 \%$ & $16,78 \%$ & $16,91 \%$ \\
\hline 4 o más & $41,53 \%$ & $38,25 \%$ & $32,65 \%$ & $32,22 \%$ & $27,52 \%$ & $25,74 \%$ \\
\hline
\end{tabular}

Fuente: Spadies 
Vol. 7

No. 1

Enero-Diciembre 2016 ISSN 1794-8231 E-ISSN 2462 - 8794

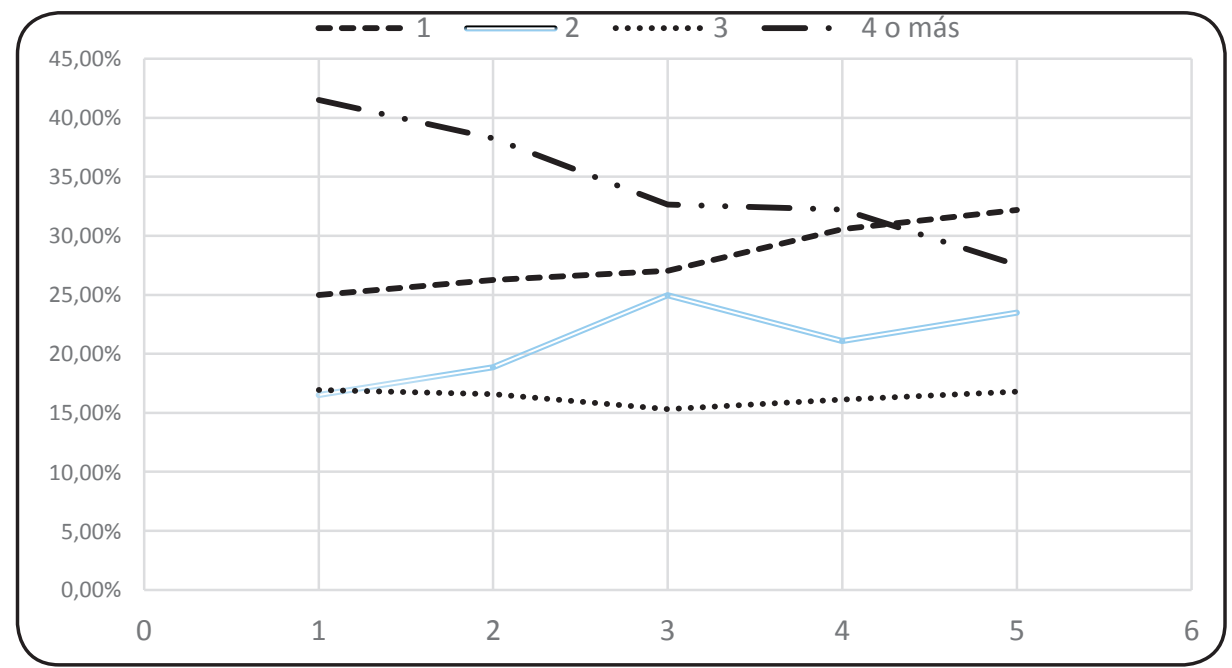

Figura 1. Número de creditos recibidos del ICETEX para estudiantes de la institución UFPS, por periodo academico de 2009-1 hasta 2011-2.

$$
\text { Fuente: SPADIES }
$$

\section{En la tabla III se observa el modelo logístico de permanencia.}

Tabla III: Modelo logístico de permanencia

\begin{tabular}{|c|c|c|c|c|c|c|}
\hline Modelo 1 & \multicolumn{4}{|l|}{ Modelo 2} & \multicolumn{2}{|l|}{ Modelo 3} \\
\hline Descripción de los parámetros estimado $\mathrm{P}$ valor & \multicolumn{4}{|c|}{$\begin{array}{l}\text { Parámetro estimado } \\
\text { Parámetro estimado }\end{array}$} & \multicolumn{2}{|c|}{$\begin{array}{l}\text { P valor Parámetro } \\
\text { P Valor }\end{array}$} \\
\hline $\begin{array}{ll}\text { Intercepto } & \\
3,5517 \quad<, 0001\end{array}$ & \multicolumn{4}{|c|}{$\begin{array}{l}3,2426 \\
3,6564<, 0001\end{array}$} & \multicolumn{2}{|l|}{$<, 0002$} \\
\hline Entre 1 y 2 SML & 0.2512 & $<, 0001$ & 0,2399 & $<, 0001$ & 0,1354 & 0,0787 \\
\hline Entre 2 y 3 SML & 0.2352 & $<, 0001$ & 0.2336 & $<0001$ & 0.0607 & 0.456 \\
\hline Entre 3 y 5 SML & 0.2800 & $<, 0001$ & 0,2957 & $<, 0001$ & 0,1324 & 0,1277 \\
\hline Dummy 5 o más SML & 0,0990 & 0,1124 & 0,1403 & 0,0221 & $-0,00802$ & 0,9345 \\
\hline Tiene Vivienda Propia & 0,0769 & 0,0392 & 0,0688 & 0,0619 & 0,0717 & 0,0908 \\
\hline Proporción de Aportantes en el hogar & $-0,2223$ & 0,0143 & $-0,2384$ & 0,0078 & $-0,1664$ & 0,1384 \\
\hline Numero de hermanos que tienen estudios superiores en curso o terminados & $-0,0001$ & 0,9931 & $-0,00225$ & 0,8737 & 0,0344 & 0,06 \\
\hline Número de personas que conforman el grupo familiar & 0,0130 & 0,2746 & 0,00902 & 0,4425 & 0,0123 & 0,4078 \\
\hline Ingenierías & $-0,8117$ & $<<, 0001$ & $-0,7175$ & $<, 0001$ & $-0,6559$ & $<, 0001$ \\
\hline Educación & $-0,5031$ & $<<, 0001$ & $-0,3898$ & $<, 0001$ & $-0,3439$ & 0,0007 \\
\hline Otra área & $-0,3814$ & $<<, 0001$ & $-0,6826$ & $<, 0001$ & $-0,6434$ & $<, 0001$ \\
\hline Icfes Bajo & $-0,4105$ & $<, 0001$ & $-0,817$ & $<, 0001$ & $-0,849$ & $<, 0001$ \\
\hline Icfes Medio & $-0,2163$ & $<, 0001$ & $-0,3985$ & $<, 0001$ & $-0,4352$ & $<, 0001$ \\
\hline Genero & 0,3514 & $<, 0001$ & 0,3557 & $<, 0001$ & 0,3985 & $<, 0001$ \\
\hline Edad & $-0,1832$ & $<, 0001$ & $-0,1832$ & $<, 0001$ & $-0,181$ & $<, 0001$ \\
\hline Edad al cuadrado & 0,0031 & $<, 0001$ & 0,00298 & $<, 0001$ & 0,00283 & $<, 0001$ \\
\hline $\begin{array}{l}\text { Interacción Dummy } 40 \% \text { más pobres por existencia de programas de } \\
\text { permanencia }\end{array}$ & & $-0,00386$ & & & 0,8017 & \\
\hline
\end{tabular}


Gladys Amanda Portilla-Pena, Yuleima Agudelo-González

\begin{tabular}{|c|c|c|c|c|c|}
\hline Modelo 1 & \multicolumn{3}{|l|}{ Modelo 2} & \multicolumn{2}{|l|}{ Modelo 3} \\
\hline Interacción Dummy $40 \%$ más pobres por dummy de programas a distancia & \multicolumn{3}{|c|}{0,0408} & \multicolumn{2}{|l|}{0,4812} \\
\hline Interacción dummy $40 \%$ más pobres por dummy de programas nocturnos & & & & $-0,1112$ & 0,0953 \\
\hline \%Concordancia & 24762 & 24762 & \multicolumn{2}{|r|}{24762} & \\
\hline Entre 1 y $2 \mathrm{Sml}$ & $-0,1058$ & 0,5335 & 0,4092 & \multicolumn{2}{|c|}{$-0,1417$} \\
\hline Entre 2 y $3 \mathrm{Sml}$ & 0,00333 & 0,9838 & $-0,0345$ & \multicolumn{2}{|c|}{0,8352} \\
\hline Entre 3 y $5 \mathrm{Sml}$ & 0,0518 & 0,7494 & 0,0123 & \multicolumn{2}{|c|}{0,9406} \\
\hline Entre 5 y $7 \mathrm{Sml}$ & 0,1493 & 0,4318 & 0,1101 & 0,5657 & \\
\hline 7 o mas Sml & $-0,2847$ & 0,3473 & $-0,327$ & 0,282 & \\
\hline Numero de años de escolaridad del padre & 0,00174 & 0,894 & 0,00228 & 0,862 & \\
\hline Numero de años de escolaridad de la Madre & 0,00706 & 0,6124 & 0,00816 & 0,5599 & \\
\hline Tiene vivienda propia & 0,0554 & 0,5865 & 0,0444 & 0,6637 & \\
\hline Proporción de aportantes en el hogar & $-0,617$ & 0,0087 & -0.5841 & 0,0133 & \\
\hline Numero de hermanos que tienen estudios superiores en curo o terminados & 0,0252 & 0,6162 & 0,0241 & 0,6323 & \\
\hline Numero de hermanos que conforman el hogar & $-0,0414$ & 0,2676 & $-0,0412$ & & \\
\hline Variables Académicas & $-0,8963$ & 0,0001 & $-0,8956$ & & \\
\hline
\end{tabular}

Enero-Diciembre 2016

\section{Fuente: Autor}

El estadístico de chi-cuadrado es la diferencia en las $-2 \log$ verosimilitudes entre el modelo final y el modelo reducido. El modelo reducido se forma omitiendo un efecto del modelo final. La hipótesis nula es que todos los parámetros de ese efecto son 0. (Tabla IV).

Tabla IV. Contrastes de la razón de verosimilitud

\begin{tabular}{|l|c|c|c|c|}
\hline \multirow{2}{*}{ Efecto } & $\begin{array}{c}\text { Criterio de ajuste del } \\
\text { modelo }\end{array}$ & \multicolumn{3}{|c|}{$\begin{array}{c}\text { Contrastes de la razón de } \\
\text { verosimilitud }\end{array}$} \\
\cline { 2 - 5 } & $\begin{array}{c}-2 \text { log verosimilitud del } \\
\text { modelo reducido }\end{array}$ & Chi-cuadrado & gl & Sig. \\
\hline Intersección &, $000^{\mathrm{a}}$ &, 000 & 0 & $\cdot$ \\
\hline año & 120,870 & 120,870 & 500 & 0,5000 \\
\hline
\end{tabular}

Fuente: autores

Este modelo (1) reducido es equivalente al modelo final ya que la omisión del efecto no incrementa los grados de libertad No obstante para predicción variable esperada el modelo no se hace óptimo p-valor 0.5 dado factores que afectan el modelo a ser analizados posteriormente. Se realiza ajuste de data según modelo y se analiza teniendo en cuenta para deserción variable académica.

$$
\begin{array}{r}
D(T)=A \times \operatorname{Exp}(\beta t) \\
D(T)=24 e^{-0.025 t}
\end{array}
$$

Donde $\mathrm{D}(\mathrm{T})$ es el porcentaje valor esperado de deserción para año según I o II semestre académico, $\beta$ variable rendimiento académico según promedio acumulado, $t$ tiempo dado en año. 


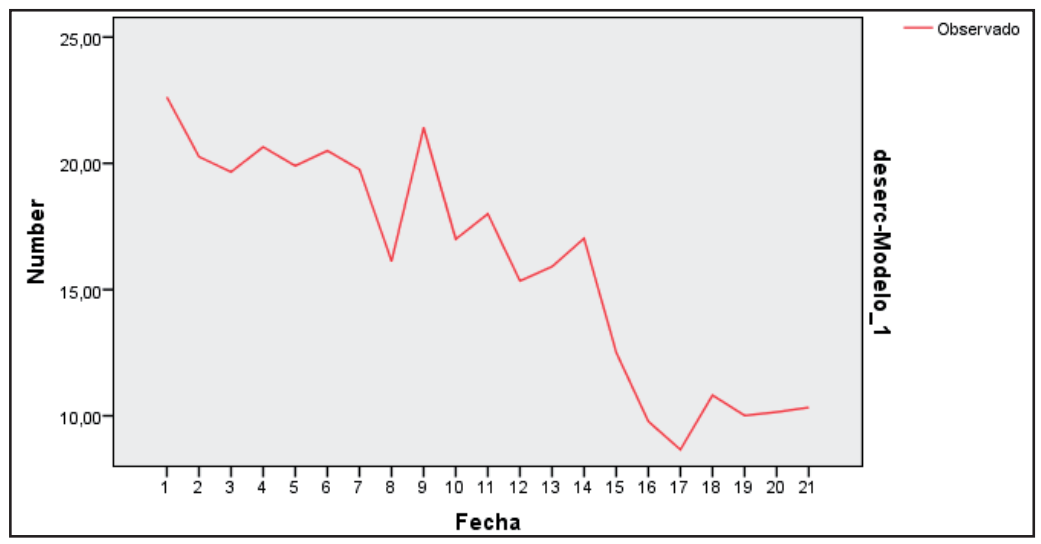

Figura 2. Deserción Observada por períodos donde fecha $\mathrm{t}=1$ representa período 2001-I Fuente: autores

Para la disminución de la deserción (tabla V) se presenta un modelo logístico de deserción elaborado de acuerdo a data de SPDIES, así como ajuste $\mathrm{D}(\mathrm{T})=24 \mathrm{e}^{-0,025 \mathrm{t}}$, esperando reducir la deserción de la institución en el año 2014 a $9.4 \%$ y a 2019 según plan de desarrollo 2011-2019 en 6.5\%. El 12,3\% del total de estudiantes matriculados en el 2009-2 no se matricularon el semestre siguiente. De igual manera resultados en el cual se muestran algunos factores críticos a atender en torno a la deserción de los estudiantes siguiendo a Gallardo y Vergel (2007). Al igual que Irwin (1985) e Ishamura (2013) para análisis de costos universidades adelantaron estudios de costo por programa de estudiantes e implementó modelo de costos del Ministerio de Educación Nacional.

\begin{tabular}{|c|c|c|c|c|c|}
\hline \multirow[b]{2}{*}{ Modelo } & \multirow{2}{*}{$\begin{array}{l}\text { Número de } \\
\text { predictores }\end{array}$} & \multirow{2}{*}{\begin{tabular}{|c|}
$\begin{array}{c}\text { Estadísticos de ajuste del } \\
\text { modelo }\end{array}$ \\
R-cuadrado estacionaria \\
\end{tabular}} & \multirow{2}{*}{\begin{tabular}{|l} 
Ljung-Box Q(18) \\
Estadísticos
\end{tabular}} & \multicolumn{2}{|r|}{ Número de valores atípicos } \\
\hline & & & & GL & \\
\hline deserc-Modelo_1 & 0 & 5782 & 12,652 & 19 & 0 \\
\hline
\end{tabular}

Variables de acción formativa sobre la existencia de programas nocturnos, a distancia y el número de programas de acción formativa existentes de prevención y acuerdos no han sido significativas. Los resultados muestran que la existencia de programas nocturnos no tiene ningún efecto en la probabilidad de permanencia, mientras que la existencia de programas de educación a distancia y programas en convenio afectan negativamente la permanencia. Por su parte, el número de programas de intervención académica tienen un efecto positivo y significativo en la probabilidad de permanencia. El ingreso familiar contribuye de forma importante a aumentar la probabilidad de acceso para los aspirantes que provienen de hogares de ingresos entre 1 y 3 salarios mínimos.

\section{Conclusiones}

$\mathrm{D}(\mathrm{T})=24 \mathrm{e}-0,025 \mathrm{t}$, esperando reducir la deserción de la institución en el año 2014 a $9.4 \%$ y a 2019 según plan de desarrollo 20112019 en $6.5 \%$.

La tasa de deserción por período para universidades es de $13.15 \%$ de 15000 estudiantes matriculados en promedio en primer semestre por año. La tasa de retención para el periodo es de $89 \%$.

Después de 10 semestres cursados el acumulado de desertores asciende a 50\% de los estudiantes que ingresan por primera vez a las universidades. 
El Plan padrino aunque permitió a muchos jóvenes estudiar entre 1 y 4 por semestre, no tuvo relevancia significativa en la deserción de las instituciones de educación básica y superior.

\section{Referencias}

Acevedo, C., Vergel, M. \& Flórez, E. (2012). Teoría de contacto aplicada al mecanismo Leva-palpador Cilíndrico, Bogotá. Editorial Universidad Nacional de Colombia.vol.1 pp.58.

Cárdenas, M., Vergel, M. \& Rincón, O. (2013). Reliquidación de matrícula. Caracterización del beneficiario en la Universidad Francisco de Paula Santander. Revista Ecomatemático, (4)1, 80-94.

Gallardo, H. \& Vergel, M. (2007). Modelación en un museo interactivo. Imaginarios. X Reunión de la RED POP y IV Taller "Ciencia, Comunicación y Sociedad". Red de popularización de la Ciencia y la Tecnología en América latina y el caribe, San José, Costa Rica.

Huertas Díaz, O., Esmeral Ariza, S., \& Sánchez Fontalvo, I. (2014). La Educación en Comunidades Indígenas; Frente a su proyecto de vida en un mundo globalizado. Revista Logos Ciencia \& Tecnología, 5(2), 232-243. doi:http://dx.doi.org/10.22335/rlct.v5i2.112

Hurtado Barrera, J. (2000). Metodología de la Investigación Holística. Venezuela: SYPALIUTC.

Irving, A. (1985). Study and information skills across the curriculum. London: Heinemann Educational.

Isaac, J.R. (2000). Identifying educational core competencies for the information age.

Ishimura, y. (2013). Information behavior and japanese students: How can an understanding of the research process lead to better information literacy? Public Services Quarterly, 9 (1), 20- 33

Martínez Lozano, J., Vergel Ortega, M., \& Zafra Tristancho, S. (2015). Validez de instrumento para medir la calidad de vida en la juventud: VIHDA. Revista Logos Ciencia \& Tecnología, 7(1), 20-28.

Martínez, J., Vergel, M. \& Zafra, L. (2015). Comportamiento Juvenil y competencias prosociales, Bogotá, Ibáñez

Reyes Ruiz, L., Castañeda Carranza, E., \& Pabón Castro, D. (2012). "Causas psicosociales de la deserción universitaria". Revista Logos Ciencia \& Tecnología, 4(1), 164-168. doi:http://dx.doi.org/10.22335/rlct. v4i1.179

Rincón, O., (2011). Evaluación de actitudes hacia la incorporación de la Calculadora Voyage 200 en las aplicaciones de las ecuaciones diferenciales de primer orden. Revista Ecomatemático, 2(1), 21-26.

Vergel, M.(2015). Autoconceptoyjuventud: el papel de los contenedores de IBERCIENCIA. Iberoaméricadivulga, Organización de Estados Iberoamericanos. Recuperado de: http://www.oei.es/historico/divulgacioncienti fica/?Autoconcepto-y-juventud-el-papel

Vergel, M., Orjuela, J. \& Martínez, J. (2014). Modelos estimados para el tiempo de permanencia de estudiantes en asignaturas de Cálculo en la Universidad Francisco de Paula Santander. En XXVIII Reunión Latinoamericana de Matemática Educativa (Relme). Universidad del Atlántico, Barranquilla, Colombia.

Vergel Ortega, M., Martínez Lozano, J., \& Zafra Tristancho, S. (2015). APPS en el rendimiento académico y autoconcepto de estudiantes de ingeniería. Revista Logos Ciencia \& Tecnología, 6(2), 198-208.

Vergel, M., Martínez, J., \& Duarte, H. (2015). Desarrollo del pensamiento matemático en
Enero-Diciembre 2016 ISSN 1794-8231 E-ISSN 2462 - 8794 PP: 141-148 
estudiantes de cálculo integral su relación con la planificación docente. Revista Científica., $3(23), 17-29$.

Vergel, M., Gallardo, H. \& Martínez, J. (2014). Factores asociados al rendimiento académico en estadística de estudiantes de administración pública. Bogotá: Colección Pedagogía Iberoamericana

Wilson, C. Et al (2011). Media and information literacy curriculum for teachers. Paris: Unesco. 192.

Wilson, E.k. y Blankenship, J.M. (2010). Integrating information literacy instruction in Upper-division writing-intensive class. Southeastern librarian, 58 (3), 20-27.

Wilson, C. (2012). Media and information literacy: pedagogy and possibilities. Comunicar, 20 (39), 15-22.

Wood, G. (2004). Academic original sin: plagiarism, the internet, and librarians. The journal of Academic Librarianship, 30 (3), 237-242.

Turizo Arzuza, M. (2014). En la búsqueda de nuevas formas de interacción sociodiscursiva en entornos virtuales de aprendizaje: El nuevo rol docente. Revista Logos Ciencia \& Tecnología, 5(2), 263-273. doi :http://dx.doi. org/10.22335/rlct.v5i2.123

Zafra, S., Martínez, J., \& Vergel, M. (2014). Indicadores para evaluar la pertinencia social en la oferta académica de programas. Revista Logos Ciencia \& Tecnología, 6(1), 165-177. doi:http://dx.doi.org/10.22335/rlct.v6i1.361 